\title{
Quantum-dot based nanothermometry in optical plasmonic recording media
}

Laura Martinez Maestro', Qiming Zhang', Xiangping Li, Daniel Jaque, and Min Gu

Citation: Appl. Phys. Lett. 105, 181110 (2014); doi: 10.1063/1.4901258

View online: http://dx.doi.org/10.1063/1.4901258

View Table of Contents: http://aip.scitation.org/toc/apl/105/18

Published by the American Institute of Physics

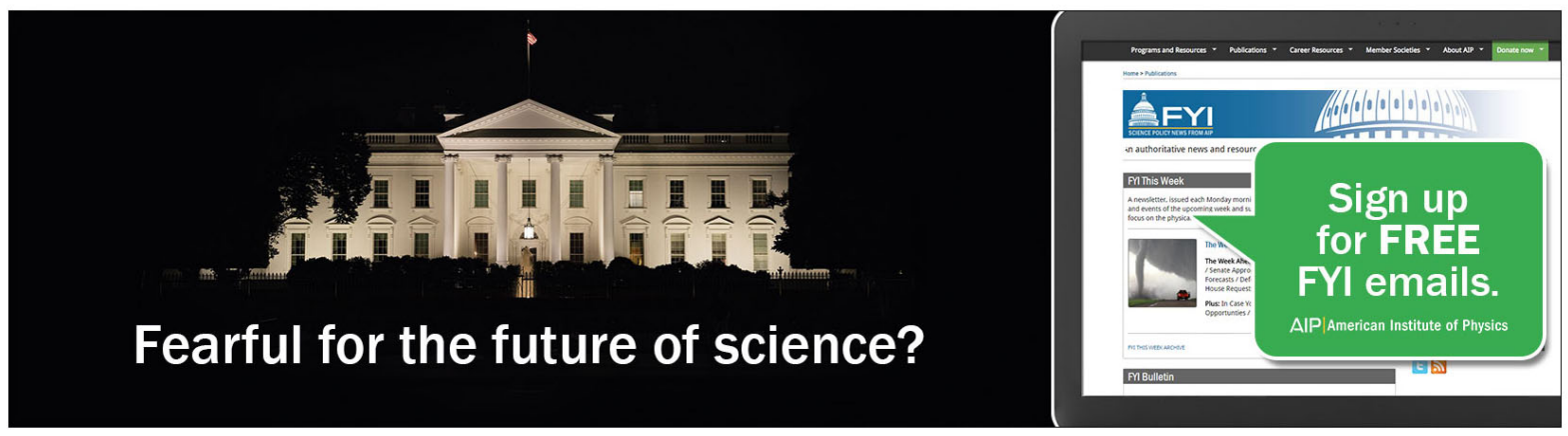




\title{
Quantum-dot based nanothermometry in optical plasmonic recording media
}

\author{
Laura Martinez Maestro, ${ }^{1,2, a)}$ Qiming Zhang, ${ }^{2, a)}$ Xiangping Li, ${ }^{2}$ Daniel Jaque, ${ }^{1}$ and Min $\mathrm{Gu}^{2}$ \\ ${ }^{1}$ Fluorescence Imaging Group, Departamento de Física de Materiales, Facultad de Ciencias Físicas, \\ Universidad Autónoma de Madrid, Madrid 28049, Spain \\ ${ }^{2}$ Centre for Micro-Photonics, Faculty of Science, Engineering and Technology, Swinburne University of \\ Technology, Hawthorn, Victoria 3122, Australia
}

(Received 2 October 2014; accepted 27 October 2014; published online 6 November 2014)

We report on the direct experimental determination of the temperature increment caused by laser
irradiation in a optical recording media constituted by a polymeric film in which gold nanorods
have been incorporated. The incorporation of CdSe quantum dots in the recording media allowed
for single beam thermal reading of the on-focus temperature from a simple analysis of the two-
photon excited fluorescence of quantum dots. Experimental results have been compared with nu-
merical simulations revealing an excellent agreement and opening a promising avenue for further
understanding and optimization of optical writing processes and media. (c) 2014

AIP Publishing LLC. [http://dx.doi.org/10.1063/1.4901258]

Gold nanorods (hereafter GNRs) are, without doubt, one of the most important building blocks in modern nanophotonics because of their unique optical, mechanical, and structural properties. ${ }^{1}$ Optical properties of GNRs are governed by the presence of the so-called surface plasmon resonances (SPRs) that correspond to a collective motion of surface charges that are induced when GNRs are excited by optical radiation of a given "resonance" wavelength (so-called surface plasmon resonance wavelength, denoted by $\left.\lambda_{\mathrm{SPR}}\right) . \lambda_{\mathrm{SPR}}$ is settled, among other factors, by the GNR shape and dimensions. ${ }^{2}$ When SPR is optically excited, there is a significant light-to-GNR energy transfer that could lead to a significant heating of the GNR itself and, consequently, of its surroundings. ${ }^{3,4}$ Because of their typically large light-to-heat conversion efficiency, ${ }^{5}$ GNRs have received a great attention as photothermal transductors capable, as an example, of efficient and non-invasive photothermal therapy of cancer tumors in animal models. ${ }^{6}$ In addition, it has been also demonstrated that how light-induced thermal loading of GNRs could be large enough to induce their partial/total melting that could result in an irreversible and drastic light-induced GNR shape change and, consequently, an irreversible change in their optical properties. ${ }^{7}$ Based on this effect, GNRs have been used for the fabrication of optical recording devices. ${ }^{8,9}$ These are based on an optical recording medium consisting by a transparent polymer containing randomly orientated GNRs. Recording is performed by scanning a modulated and tightly focused laser beam in such a way that its absorption for GNRs of adequate shape and orientation induces a fast and well localized temperature rise in the GNRs. ${ }^{10}$ For sufficiently high laser energies, the absorbing GNRs will heat up to above the threshold melting temperature and transform their shapes into shorter rods or spherical particles. ${ }^{8}$ This results in a well spatially localized population depletion of GNRs with a certain aspect ratio and orientation. Reading was performed by scanning a low power laser beam and measuring, for different polarization and wavelengths, the spatial distribution of the SPR-assisted two photon

${ }^{a}$ L. M. Maestro and Q. Zhang contributed equally to this work. luminescence generated by non-melted GNRs. Based on this approach, storage densities as large as $1.1 \mathrm{Tbits} / \mathrm{cm}^{3}$ have been, indeed, demonstrated. ${ }^{10}$ It is crucial to reduce the cross talks in such an approach for high density information storage to manipulate the heating up of corresponding GNRs only by their deterministic polarization states and resonance wavelengths. Consequently, full control and future optimization of the writing parameters require an accurate knowledge of the local temperature increment induced in the recording medium by the laser irradiation. Despite of its interest, there is no, up to know, any work reporting on an experimental determination of the temperatures achieved during laser writing of optical memories. Writing parameters have been adjusted in the past accordingly to numerical simulations whose correctness (agreement between simulated and real temperature increment) remains, still, to be checked. ${ }^{11}$ Such a corroboration would require a real time measurement of the laser induced temperature at the focus of the writing laser that is far from being an easy task. A possible approach could be the use of the so-called Luminescence Nanothermometers (LNthMs) that consist on nanoparticles (NPs) that show strongly temperature dependent luminescence. ${ }^{12}$ A great variety of luminescent NPs have been demonstrated to be capable of high resolution luminescence thermal sensing. ${ }^{13}$ Special mention has to be done to the use of semiconductor quantum dots (QDs) as two-photon excited LNthMs based on the temperature induced spectral shift of their luminescence band. ${ }^{14,15}$ For the particular case of CdSe-QDs, their luminescence has been found to suffer from a linear red-shift with temperature at a rate close to $0.1 \mathrm{~nm} /{ }^{\circ} \mathrm{C}$, this allowing in the past for high resolution thermal sensing and imaging in different systems including living cells and microfluidics. ${ }^{14,16,17}$ Very interestingly, CdSeQDs are efficiently excited by $800 \mathrm{~nm}$ laser pulses, the same used for optical recording. Therefore, CdSe-QD based LNthMs seem to be especially suitable for fluorescence thermal sensing in optical recording media by using a single laser beam. Nevertheless, and despite of its potential interest, the use of CdSe-QDs for thermal measurements optical recording media has not been attempted up to now. 
In this work, we have incorporated CdSe-QDs into an optical recording medium consisting of GNR dispersed into a polymer film. The writing laser wavelength (close to $800 \mathrm{~nm}$ and matching $\left.\lambda_{\mathrm{SPR}}\right)$ also promotes the two-photon excitation of the temperature sensitive visible luminescence of CdSe-QDs. This fact has allowed us to determine the onfocus temperature of the optical recording media by using a simple single beam excitation set-up. Experimental results have been compared to numerical simulations. The influence that the polarization state of the laser writing beam polarization has on the thermal loading has been also investigated and discussed.

Figure 1 schematically shows the experimental set-up used in this work. The recording medium consists of a thick film of a $20 \mu \mathrm{m}$ thick polyvinyl alcohol (PVA) polymer deposited on a microscope slide by spin coating. ${ }^{10}$ As explained in the introduction, optical recording is based on the laser-induced melting of GNRs that were incorporate into the film. In this work, the GNR concentration was set to 72 particles per $\mu \mathrm{m}^{3}$, this leading to an average distance between adjacent GNRs close to $240 \mathrm{~nm}$. GNRs were synthesized by the wet chemical synthesis. ${ }^{18}$ The dimensions of the synthesized GNRs were, on average, $15 \mathrm{~nm}$ in width and $45 \mathrm{~nm}$ in length. The surface plasmon resonance of the assynthesized GNRs was found to be centered at $800 \mathrm{~nm}$. This was only slightly modified by the incorporation into the polymer film as can be appreciated in Figure 1 that includes the extinction coefficient of the optical recording medium used all along this work, denoting the presence of the plasmon resonance of GNRs at around $800 \mathrm{~nm}$. Optical excitation of GNRs was performed by using a $100 \mathrm{fs}, 80 \mathrm{MHz}$ mode-locked Ti:sapphire laser tuned to $800 \mathrm{~nm}$ that was focused into the recording film by using a 0.75 NA microscope objective, leading to a laser spot of $1.3 \mu \mathrm{m}$ in diameter inside the recording media. Although optical recording can be also achieved by using continuous wave lasers, in this work, we decided to use fs laser pulses in order to excite the two-photon fluorescence of CdSe-QDs. Since numerical simulations of laser induced thermal loading in recording media use optical parameters of non-modified GNRs, i.e., describe the pre-melting state, special care was taken to keep laser power well below the "melting limit." This would ensure a minimal morphological modification of GNRs and, hence, a minimal variation of their optical properties. All along our experiments, laser power was only varied between 1 and 5
$\mathrm{mW}$. For the purpose of thermal sensing, CdSe-QDs were also incorporated into the polymer before film deposition. The concentration of CdSe-QDs was set to be close to 10 particles per $\mu \mathrm{m}^{3}$, being this a concentration ensuring good signal-to-noise ratio measurements of their fluorescence (required for accurate temperature determination from the analysis of fluorescence band). The two-photon excitation of CdSe-QDs consists of a broad band extending from $700 \mathrm{~nm}$ up to $950 \mathrm{~nm}$ so that, as commented previously, the $800 \mathrm{~nm}$ laser pulses used for plasmonic excitation of GNRs also stimulated the two-photon excited red luminescence of CdSe-QDs. ${ }^{19}$ This fact is schematically drawn in Figure 1. The CdSe-QD fluorescence was collected by the same microscope objective used for $800 \mathrm{~nm}$ laser pulses focusing and was spectrally analyzed by using a high resolution fibercoupled spectrometer.

Typical fluorescence spectra obtained in our experimental conditions are included in Figure 2(a) as obtained for two different $800 \mathrm{~nm}$ laser powers. The characteristic broadband emission of CdSe-QDs is clearly observed in both cases with not relevant contribution of the two-photon excited fluorescence of GNRs that would lead to a broad band extending from $500 \mathrm{~nm}$ up to $700 \mathrm{~nm}$, not observed in Figure 2(a). Data included in Figure 2(a) evidence as a strong dependence of the CdSe-QD luminescence on the $800 \mathrm{~nm}$ laser power. When laser power is increased, remarkable red-shift and luminescence quenching are found to occur. According to the previous works, ${ }^{15}$ these two facts are a clear signature of a relevant temperature increment at the $800 \mathrm{~nm}$ laser focus inside the optical recording media. The magnitude of such laser induced heating can be, indeed, estimated from a simple spectral analysis of the CdSe-QDs luminescence. In colloidal solutions, the emission peak of CdSe-QDs suffers from a temperature-induced red-shift at a rate on $0.1 \mathrm{~nm} /$ ${ }^{\circ} \mathrm{C},{ }^{17}$ being this value determined by the combination of several factors (such as QD thermal expansion and temperature dependence of band gap) that could be modified when CdSeQDs are embedded in a polymer medium. ${ }^{20}$ QDs have been tested to be reversible and reliable nanothermometers up to temperatures as large as $80^{\circ} \mathrm{C}$. Thus, the determination of the on-focus temperature from the spectral analysis of the CdSe-QD luminescence requires a previous calibration. This is included in Figure 2(b) in which the CdSe-QD emission wavelength shift is plotted as a function of the optical recording media temperature. A clear linear relation is

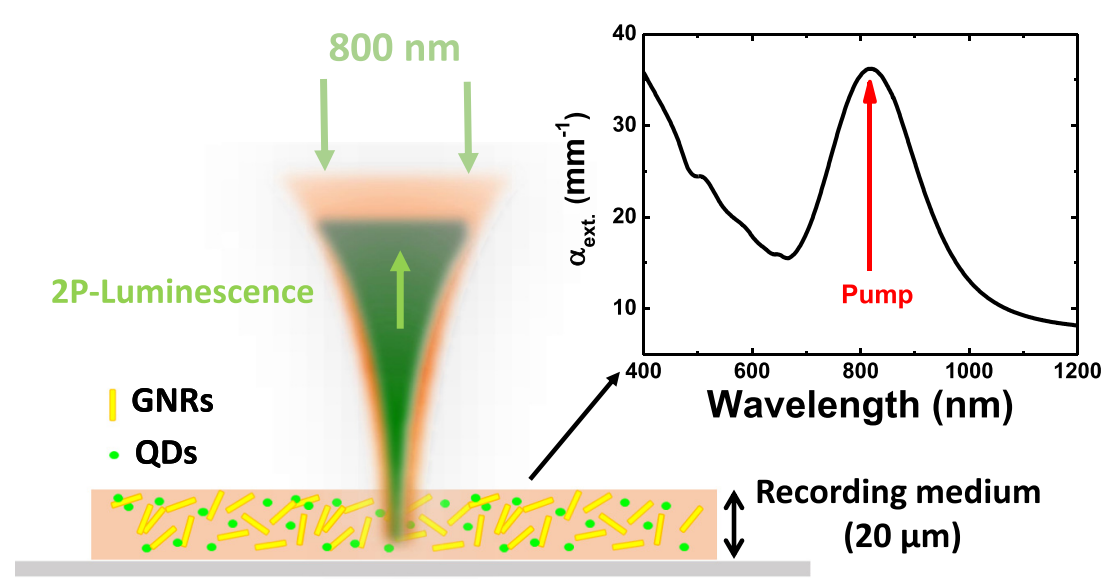

FIG. 1. Schematic representation of the experimental set-up used in this work for thermal sensing inside optical data storage media. As an inset can be seen, the characteristic extinction spectra corresponding to the GNR based recording media in which CdSeQDs have been incorporated as nanothermometers. 

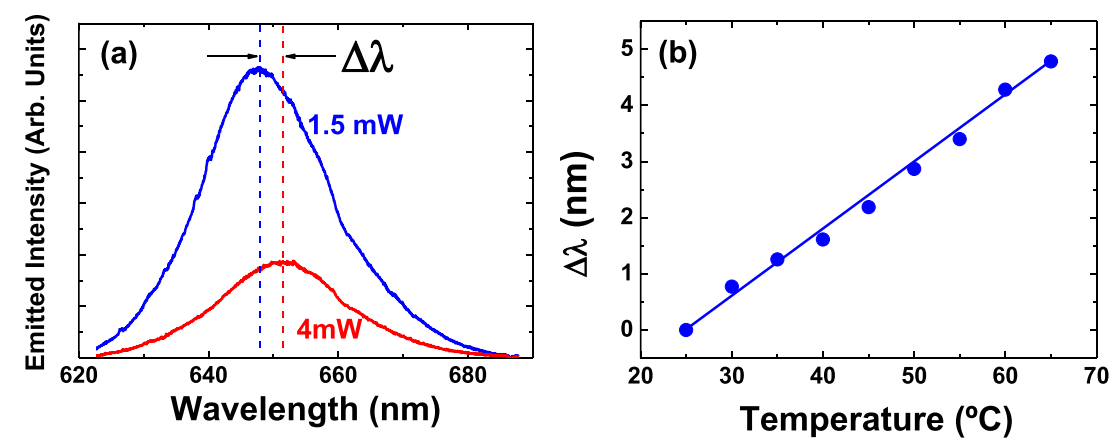

FIG. 2. (a) Characteristic two-photon emission spectra obtained from the PVA recording medium and corresponding to CdSe-QDs as obtained under high/low laser power $(800 \mathrm{~nm})$ excitation. The spectral shift reveals a remarkable increase in the on-focus temperature due to laser induced plasmonic heating. (b) Wavelength shift versus temperature calibration curve of the CdSe-QDs dispersed in the recording media. obtained, this being a desirable feature for thermal sensing purposes as it ensures a constant sensitivity in the whole range. The linear fit of experimental data concludes that the thermal induced spectral shift rate of CdSe-QDs in the polymer is $0.11 \mathrm{~nm} /{ }^{\circ} \mathrm{C}$, i.e., virtually the same as that previously reported for CdSe-QDs in a colloidal solution. ${ }^{15,17}$

Based on the calibration curve shown in Figure 2(b), it is now possible to determine the $800 \mathrm{~nm}$ laser power dependence of the on-focus temperature induced inside the recording media. For this purpose, the laser power dependence of the peak wavelength was determined and translated into temperature units using the linear calibration curve of Figure 2(b). The on-focus temperature experimentally determined for different $800 \mathrm{~nm}$ laser powers has been included in Figure 3(a). At this point, several points should be noted. First, the use of a single beam configuration ensures that the measured temperature is that exactly corresponding to the temperature induced by the absorption of GNRs at the focus of the writing laser. Second, the $800 \mathrm{~nm}$ beam intensity follows a Gaussian distribution and, consequently, the induced temperature is expected to vary within the laser spot. Since we are also collecting the whole CdSe-QD emission generated from the irradiated spot, the temperature estimated from the CdSe-QD luminescence and included in Figure 3(a) corresponds to an average temperature over the whole excitation area $\left(1.3 \mu \mathrm{m}^{2}\right)$. At this point, it should be also noted that despite the nanoscale nature of the heating sources (GNRs) we expect, in our experimental conditions, to have a smooth temperature profile at the microscale. According to the formalism developed by Baffou et al., in our experimental conditions, we estimate that for a given GNR, the temperature increment induced by adjacent GNRs is larger than the temperature increment caused by the GNR itself. ${ }^{3,21}$ In these conditions, the measured average temperature would be very close to the actual GNR temperature, the parameter of interest in 5D optical recording. In any case, a clear linear relation between the average on-focus temperature increment and the $800 \mathrm{~nm}$ laser writing power has been found. Such a linear relation is a clear indicator that, for the laser powers used in this work, non-linear processes (such as contribution of two-photon excitations, laser self-focusing, and irreversible laser induce GNR melting) are not playing a relevant role. Additionally, we have performed the same experiments in a recording film without GNRs, i.e., only containing CdSe-QDs. This sample was treated as a "control" medium in order to check for the possible contribution of the CdSeQDs nanothermometers to the laser induced thermal loading. Results obtained in this "control medium" have been also included in Figure 3(a). As can be observed, no local heating was noted in this case. This clearly evidences that the contribution of CdSe-QDs to the laser induced heating in our recording media can be neglected. We should also note that the on focus temperature determined in this work corresponds to a "time-average" temperature. Under pulse excitation and in some experimental conditions, temperature could

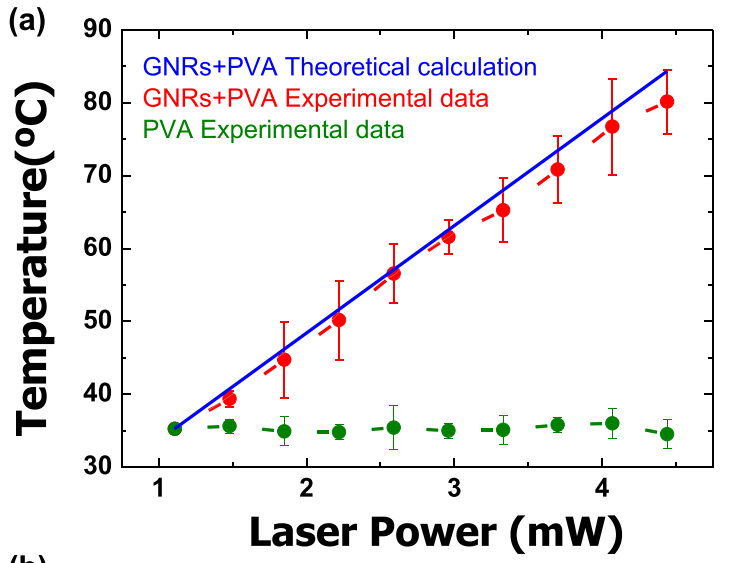

(b)
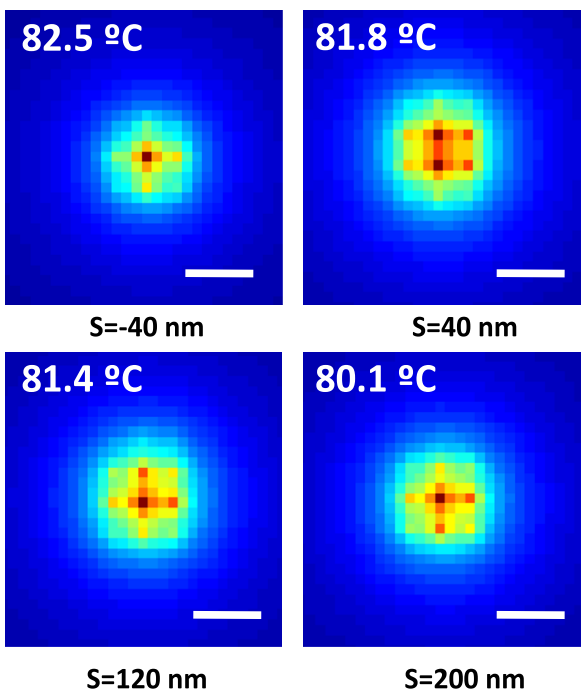

FIG. 3. (a) On focus temperature induced by the $800 \mathrm{~nm}$ laser excitation beam inside the recording media as a function of laser power. Red dots are experimental data, while green correspond to the "control" experiment (in absence of GNRs). The blue line corresponds to data extracted from bidimensional simulations. (b) Numerical simulations of the temperature distribution in the surroundings of the $800 \mathrm{~nm}$ writing laser as obtained for a laser power of $4 \mathrm{~mW}$. Each pattern corresponds to a different distance between the $800 \mathrm{~nm}$ beam axis and the closest GNR (denoted by S). The average temperature obtained in each case is also included. Scale bar is in all the cases $500 \mathrm{~nm}$. 
show relevant time-variations following the laser pulse train. $^{21,22}$ Nevertheless, in our particular case, we have estimated a characteristic time for temperature variations in the PVA medium to be close to $10 \mu \mathrm{s} .^{23}$ This is much longer than the time between consecutive laser pulses (close to $10 \mathrm{~ns})$. Consequently, the temperature inside the laser spot is expected to be steady even though the illumination is pulsed so that the time average (measured) temperature coincides with the instantaneous temperature inside the medium.

Experimental results can be now compared to the numerical simulations. Figure 3(b) includes the numerical simulations of the temperature distribution in the surroundings of the $800 \mathrm{~nm}$ writing laser as obtained for a $800 \mathrm{~nm}$ laser power of $4 \mathrm{~mW}$. The simulation method is discussed in our previous publication. ${ }^{24}$ As commented before, in our experimental conditions, the averaged distance between GNRs is close to $240 \mathrm{~nm}$, i.e., comparable to the $800 \mathrm{~nm}$ beam radius. This means that a discrete/reduced number of GNRs can be found at the laser focus. In these conditions, the distance between GNR and $800 \mathrm{~nm}$ laser beam axis could be a critical parameter. In order to check of this possibility, we have explored possible influence of the relative distance between GNR and writing beam axis on the laser induced temperature pattern by performing different numerical simulations in which the position of the $800 \mathrm{~nm}$ beam axis and the closest GNR has been varied. Results are summarized in the different thermal patterns included in Figure 3(b). Simulations correspond to a $800 \mathrm{~nm}$ laser power of $4 \mathrm{~mW}$. For each simulation, the average temperature over the laser irradiated sea has been calculated and it is indicated in each case. It has been found that the relative position between the $800 \mathrm{~nm}$ laser axis and the closest GNR has a minimal influence in the calculated average temperature. Indeed, it has been found that the different relative position of GNR assumed in the simulations of Figure 3(b) lead to variations in the average temperature not larger than $0.67 \%$. Figure $3(\mathrm{a})$ includes as a solid line the power dependence of the on-focus average temperature increment as obtained from numerical simulations by averaging between the results obtained for different position of GNRs inside the laser beam. As can be observed, a quite good agreement between modeling results and experimental data has been obtained. This fact fully validates the use of the thermal modeling procedures used in this work for a full understanding and future optimization of the laser writing in optical recording media.

The overall temperature increment within the focal volume can be manipulated by varying the polarization states of the incident illumination. Figure 4 shows the comparison of the focal temperature measurement between circular, linear, and radial polarization states. The reasonable agreement between experimental and theoretical result further confirms the validity of our model. Among the three employed polarization states, the radial polarization excitation exhibits the relatively lower temperature rising due to a relative larger spot size, which indicates a less heat accumulation at the same input power of $4 \mathrm{~mW}$ during the laser writing process. This result implies the advantage of radial polarization for high density multiplexed optical recording ${ }^{9}$ by preventing the un-wanted melting of the GNRs away from the deterministic polarization states. This is of special relevance when

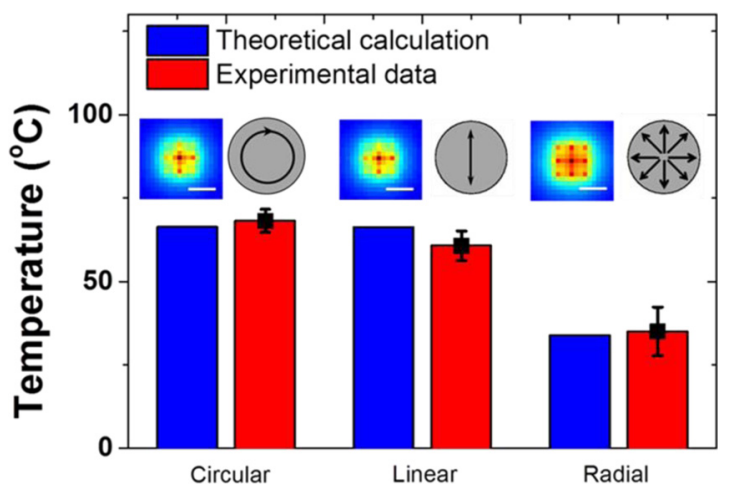

FIG. 4. Experimental and theoretical result of the on focus temperature induced by the $800 \mathrm{~nm}$ laser excitation with circular, linear, and radial polarization at a power of $4 \mathrm{~mW}$ inside the recording media. Inset: Numerical simulations of the temperature distribution in the surroundings of the $800 \mathrm{~nm}$ writing laser as obtained for a laser power of $4 \mathrm{~mW}$. Scale bar: $500 \mathrm{~nm}$.

optical recording is based on the combination of radial and azimuthal polarized beams. ${ }^{9}$

In summary, we have demonstrated that it is possible to monitor, in real time, the temperature of a GNR based optical recording media during laser irradiation by the incorporation of quantum dot luminescent nanothermometers. The ability of $800 \mathrm{~nm}$ femtosecond laser pulses for simultaneous excitation of the surface plasmon resonance of GNRs and of the thermal sensitive luminescence of CdSe-QDs allowed for simultaneous local heating and thermal reading of the optical recording medium by using a simple single-beam experimental set-up. Experimental data and numerical simulations were found to be in excellent agreement validating in this way previous assumptions and parameter optimizations done on the basis of numerical simulations. The possibility here demonstrated of getting a direct measurement of local temperature during optical recording opens exciting avenues towards a full understanding and further optimization of the optical storage devices.

This work has been supported by the Spanish Ministerio de Inovacion y Competitividad (MAT2010-16161 and MAT2013-47395-C4-1-R). L. M. Maestro thanks the Spanish Ministerio de Economía y Competitividad (MINECO) for a FPI Grant. Min Gu, Xiangping Li, and Qiming Zhang acknowledge the support from the Australian Research Council (ARC) Laureate Fellowship program (FL100100099). L. M. Maestro acknowledges the ARC Laureate Fellowship project for its support during her study in Swinburne University.

${ }^{1}$ X. H. Huang, S. Neretina, and M. A. El-Sayed, Adv. Mater. 21(48), 4880 (2009); Y.-Y. Yu, S.-S. Chang, C.-L. Lee, and C. R. C. Wang, J. Phys. Chem. B 101(34), 6661 (1997).

${ }^{2}$ S. Eustis and M. A. El-Sayed, Chem. Soc. Rev. 35(3), 209 (2006); S. Link and M. A. El-Sayed, J. Phys. Chem. B 103(40), 8410 (1999).

${ }^{3}$ H. H. Richardson, M. T. Carlson, P. J. Tandler, P. Hernandez, and A. O. Govorov, Nano Lett. 9(3), 1139 (2009).

${ }^{4}$ G. Baffou, R. Quidant, and C. Girard, Appl. Phys. Lett. 94(15), 153109 (2009).

${ }^{5}$ L. M. Maestro, P. Haro-González, J. G. Coello, and D. Jaque, Appl. Phys. Lett. 100(20), 201110 (2012); L. M. Maestro, P. Haro-González, A. Sánchez-Iglesias, L. M. Liz-Marzán, J. G. Sole', and D. Jaque, Langmuir 30(6), 1650 (2014). 
${ }^{6}$ X. Huang, I. H. El-Sayed, W. Qian, and M. A. El-Sayed, J. Am. Chem. Soc. 128(6), 2115 (2006); E. B. Dickerson, E. C. Dreaden, X. H. Huang, I. H. El-Sayed, H. H. Chu, S. Pushpanketh, J. F. McDonald, and M. A. ElSayed, Cancer Lett. 269(1), 57 (2008); L. Jing-Liang and G. Min, IEEE J. Sel. Top. Quantum Electron. 16(4), 989 (2010).

${ }^{7}$ M. A. El-Sayed, Acc. Chem. Res. 34(4), 257 (2001); S. Link, C. Burda, B. Nikoobakht, and M. A. El-Sayed, J. Phys. Chem. B 104(26), 6152 (2000); H. Petrova, J. P. Juste, I. Pastoriza-Santos, G. V. Hartland, and L. M. LizMarzan, Phys. Chem. Chem. Phys. 8(7), 814 (2006).

${ }^{8}$ M. Gu, X. Li, and Y. Cao, Light: Sci. Appl. 3, e177 (2014).

${ }^{9}$ X. P. Li, T.-H. Lan, C.-H. Tien, and M. Gu, Nat. Commun. 3, 998 (2012).

${ }^{10} \mathrm{P}$. Zijlstra, J. W. Chon, and M. Gu, Nature 459(7245), 410 (2009).

${ }^{11}$ J. Wang, Y. Chen, X. Chen, J. Hao, M. Yan, and M. Qiu, Opt. Express 19(15), 14726 (2011).

${ }^{12}$ C. D. S. Brites, P. P. Lima, N. J. O. Silva, A. Millan, V. S. Amaral, F. Palacio, and L. D. Carlos, Nanoscale 4(16), 4799 (2012); D. Jaque and F. Vetrone, ibid. 4(15), 4301 (2012).

${ }^{13}$ F. Vetrone, R. Naccache, A. Zamarron, A. J. de la Fuente, F. SanzRodriguez, L. Martinez Maestro, E. Martin Rodriguez, D. Jaque, J. G. Sole, and J. A. Capobianco, ACS Nano 4(6), 3254 (2010).
${ }^{14}$ L. M. Maestro, E. M. Rodriguez, F. S. Rodriguez, M. C. I. la Cruz, A. Juarranz, R. Naccache, F. Vetrone, D. Jaque, J. A. Capobianco, and J. G. Sole, Nano Lett. 10(12), 5109 (2010).

${ }^{15} \mathrm{~S}$. Li, K. Zhang, J. M. Yang, L. W. Lin, and H. Yang, Nano Lett. 7(10), 3102 (2007).

${ }^{16}$ D. Choudhury, D. Jaque, A. Rodenas, W. T. Ramsay, L. Paterson, and A. K. Kar, Lab Chip 12(13), 2414 (2012).

${ }^{17}$ J.-M. Yang, H. Yang, and L. Lin, ACS Nano 5(6), 5067 (2011).

${ }^{18}$ B. Nikoobakht and M. A. El-Sayed, Chem. Mater. 15(10), 1957 (2003).

${ }^{19}$ L. M. Maestro, E. M. Rodriguez, F. Vetrone, R. Naccache, H. L. Ramirez, D. Jaque, J. A. Capobianco, and J. G. Solé, Opt. Express 18, 23544 (2010).

${ }^{20}$ A. Olkhovets, R. C. Hsu, A. Lipovskii, and F. W. Wise, Phys. Rev. Lett. 81(16), 3539 (1998).

${ }^{21}$ G. Baffou, P. Berto, E. B. Ureña, R. Quidant, S. Monneret, J. Polleux, and H. Rigneault, ACS Nano 7(8), 6478 (2013).

${ }^{22}$ G. Baffou and H. Rigneault, Phys. Rev. B 84(3), 035415 (2011).

${ }^{23}$ S. Serak, A. Kovalev, A. Agashkov, H. F. Gleeson, S. J. Watson, V. Reshetnyak, and O. Yaroshchuk, Opt. Commun. 187(1-3), 235 (2001).

${ }^{24} \mathrm{Md}$ Azim Ullah, Xiangping Li, Xueming Cheng, Xiaojian Hao, Yahui Su, Jianshe Ma, and Min Gu, Opt. Express 20(22), 24516 (2012). 